\title{
Expanding the genotype-phenotype spectrum of autosomal recessive Charcot-Marie-Tooth disease: A novel PLEKHG5 gene mutation
}

\author{
${ }^{1}$ Özlem YAYICI KÖKEN $M D,{ }^{2}$ Ülkühan ÖZTOPRAK $m D,{ }^{3}$ Vehap TOPÇU $M D$, \\ ${ }^{3}$ Büşranur ÇAVDARLI ${ }_{M D},{ }^{4}$ Çağrı Mesut TEMUÇIN ${ }_{m D},{ }^{5}$ Üstün AYDINGÖZ ${ }_{M D}$, \\ ${ }^{6}$ Özge DEDEOĞLU $M D,{ }^{7}$ Hülya KAYILIOĞLU $M D,{ }^{2}$ Deniz YÜKSEL $M D$
}

${ }^{1}$ Ankara City Hospital, Children's Hospital, Dept of Pediatric Neurology, Ankara; ${ }^{2}$ University of Health Sciences, Dr. Sami Ulus Training and Research Hospital, Dept of Pediatric Neurology, Ankara; ${ }^{3}$ Ankara City Hospital, Dept of Medical Genetics, Ankara (previously Numune Training and Research Hospital); ${ }^{4}$ Hacettepe University, Department of Neurology, Ankara; ${ }^{5}$ Hacettepe University, Department of Radiology, Ankara; ${ }^{6}$ Mardin State Hospital, Department of Pediatric Neurology, Mardin; ${ }^{7}$ Muğla Sitkı Koçman University, Research and Training Hospital, Muğla, Turkey

\begin{abstract}
Autosomal recessive intermediate Charcot Marie Tooth (CMT) disease type C is a very rarely-seen neurogenetic disorder. Homozygous or compound heterozygous mutation in the Pleckstrin homology domain-containing family $\mathrm{G}$ member 5 (PLEKHG5) gene on chromosome $1 \mathrm{p} 36$ was recently reported in patients with CMT. From the first description of the disease to date, almost 40 different variants associated with the PLEKHG5 gene were identified. Here, we present an adolescent girl who was thought initially to be myopathy because of progressive proximal muscle weakness. The electrophysiologic study revealed axonal sensory and motor neuropathy with some demyelinating features. She was diagnosed with autosomal recessive inheritance, intermediate CMT disease type C with a novel homozygous mutation in the PLEKHG5 gene in clinical exome sequencing as c.1600$2 A>G$ by next-generation sequencing. We describe here the novel mutation in the PLEKHG5 gene and the genotype-phenotype correlation.
\end{abstract}

Keywords: PLEKHG5 gene, Charcot-Marie-Tooth disease, autosomal recessive, peripheral neuropathy

\section{INTRODUCTION}

Pleckstrin homology domain-containing family $\mathrm{G}$ member 5 (PLEKHG5) plays a major role predominantly in the peripheral nervous system, which regulates plasticity, synapse formation, dendrite growth, neuronal shape, and neuronal survival. ${ }^{1}$ The mutations in PLEKHG5 were reported in 2 main phenotypes, lower motor neuron disease (LMND) and intermediate Charcot-Marie-Tooth (CMT) disease. ${ }^{2,3}$ Sensory neuropathy is the main discriminator between the 2 entities. To date, compound heterozygous and homozygous mutations in the PLEKHG5 gene related to CMT disease were reported. Motor and sensory neuropathy in electrophysiological studies and neurological examination are characterized by progressive distal $>$ proximal symmetric muscle atrophy and weakness, initially presenting as distal muscle weakness of the lower limbs, and foot and spine deformity were observed in almost all of the patients. ${ }^{3-5}$ In this report, the case of a Turkish girl who was diagnosed with autosomal recessive (AR) intermediate CMT disease type-C (ARCMTRIC) with a novel homozygous mutation in the PLEKHG5 gene is presented.

This is a case report of a female patient in clinical follow-up at the Department of Pediatric Neurology, University of Health Sciences (SBU), Dr. Sami Ulus Training and Research Hospital, Ankara, Turkey. The patient and her legal guardians gave written consent for the publication of demographic, clinical, and laboratory data. The genetic study was performed at the Department of Medical Genetics, University of Health Sciences,

Address correspondence to: Özlem Yayıcı Köken, MD, Pediatric Neurologist, Ankara City Hospital, Children's Hospital, Department of Pediatric Neurology, Ankara Sehir Hastanesi, Cocuk Hastanesi Üniversiteler Mahallesi 1604. Cadde No: 9 Çankaya, Ankara, Turkey. Tel: +905307624200, Email: drozlemkoken@ gmail.com

Date of Submission: 7 Januray 2021; Date of Acceptance: 21 May 2021

https://doi.org/10.54029/2021jmr 
Numune Training and Research Hospital, Ankara, Turkey, and informed consent for the genetic study was obtained from the patient and her parents. DNA was extracted using the peripheral blood of the patient. Next-generation sequencing was used with the Illumina Trusight One Panel (Trusight One, Illumina, USA). Electromyography (EMG) was performed at the Department of Neurology, Hacettepe University, Ankara, Turkey. Lowerlimb magnetic resonance imaging (MRI) was performed using T1-, T2-, and STIR-weighted spin echo at the Department of Radiology of Hacettepe University.

\section{CASE REPORT}

This 8-year-old female was referred to the Department of Pediatric Neurology due to frequent falls and difficulty in climbing stairs. Symptoms had been present since the previous year but were mildly progressive. She was born at term without complications from consanguineous parents. Her prenatal, natal, and postnatal history was unremarkable. The parents reported that she had normal motor and mental development compared with her peers during infancy and childhood. The family history was unremarkable for neuromuscular diseases. On neurological examination at referral, she was weak prominently in the limb-girdle muscle. While she could barely walk on her toes and heels, she had more obvious difficulty standing up from a sitting position. Her Gowers' sign test after a duration of $10 \mathrm{~s}$ were positive for weakness in her limb-girdle muscles. She was able to walk without support. Deep tendon reflexes were absent. Her sensory system was intact with the evaluation of touch, position, and vibration. Creatine kinase (CPK) levels were slightly elevated at $400 \pm 120 \mathrm{IU} / \mathrm{L}$ (normal range of CPK: 50-150 IU/L). Metabolic tests, such as tandem mass, urine, and serum organic acids, lactate, and pyruvate levels, were all within normal limits.

During follow-up, motor regression was observed with Gowers' sign test was positive with a longer duration. At her last visit, at the age of 10 years, she could not stand up from a sitting position without support. Neurological examination revealed muscle weakness and atrophy of bilateral distal muscles, predominantly in the lower limbs. Thoracolumbar scoliosis $(25$ degrees) was observed. Cerebellar and pyramidal signs and cognitive functions were within normal limits. Her cardiac evaluation, including an electrocardiogram and echocardiogram, was within normal limits. Eye examination and hearing tests were in normal ranges.

\section{Electromyography}

At her first electrophysiological examination at 8 years of age, motor nerve conduction studies (NCSs) and F-wave studies were performed for the right tibial, ulnar, and peroneal nerves, and sensory NCSs were performed for the right median, ulnar, and bilateral sural nerves using standard techniques. F-wave latencies were significantly prolonged in the median and tibial motor nerves and could not be obtained for the ulnar and peroneal nerves. The ulnar and median motor and sensory nerve conduction velocities were slow (Table 1). ${ }^{6}$ The sural nerve sensory action potentials could not be obtained. Slight chronic neurogenic findings were determined upon needle EMG examination of the tibialis anterior muscle, and the motor unit action potentials had slightly long duration and large amplitude. The first electrophysiological study suggested sensory and motor intermediate polyneuropathy with moderate demyelinating features.

At the second electrophysiological examination at 9 years of age, F-wave abnormalities of the median and tibial nerves remained unchanged, while F-waves could be obtained for the peroneal and ulnar nerves, but with prolonged latencies (Table 1). The ulnar and median motor, and ulnar sensory nerve conduction velocities were within normal ranges, while the median sensory nerve conduction velocity was still slow. ${ }^{6}$ Bilateral sural nerve sensory action potentials could be obtained, but with very low amplitudes. There were both significant active (fibrillation potentials and positive sharp waves) and chronic neurogenic findings for the tibialis anterior, iliopsoas, biceps, and first dorsal interosseous muscles with needle EMG examination. There were myokymic discharges in the biceps muscle. The second electrophysiological study suggested a predominantly axonal sensory and motor polyneuropathy and some demyelinating features.

\section{Magnetic resonance imaging}

Her brain MRI was normal. The MRI of the lower limbs showed a Goutallier classification of 1 degree of fatty changes in both the anterior and medial compartment muscles. Her bone structure was normal. The thickness of the subcutaneous fat tissue was significantly increased (Figure 1). 


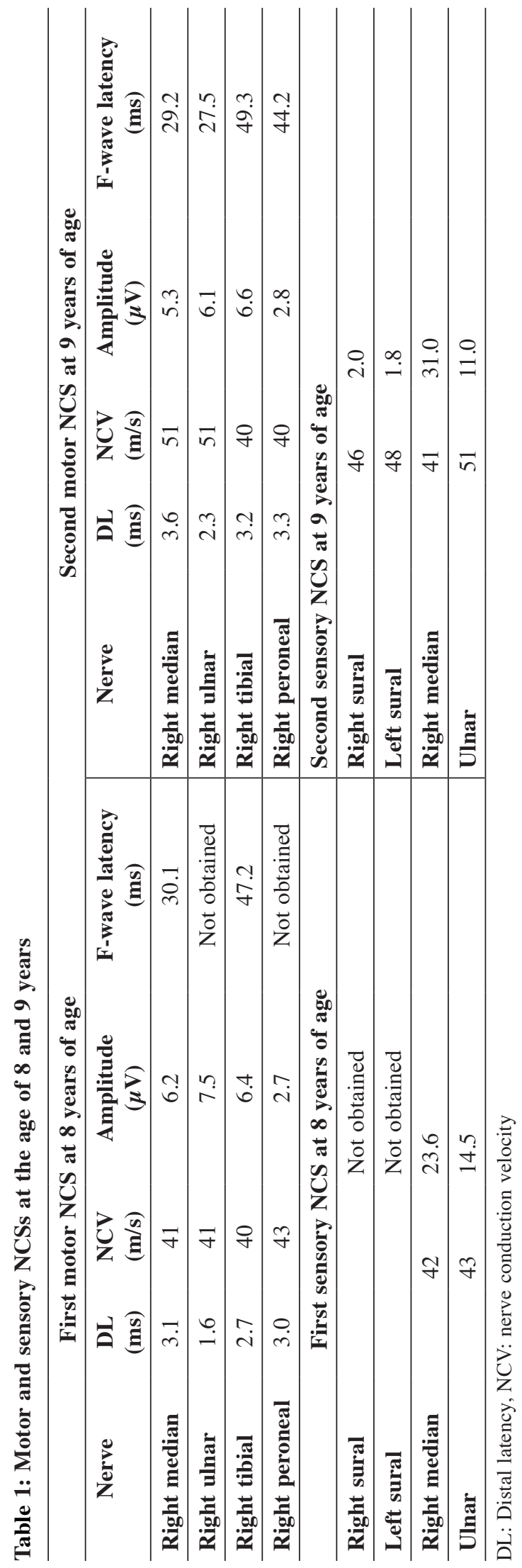

\section{Genetic study}

Clinical exome sequencing performed with TruSight $^{\mathrm{TM}}$ One Sequencing Panel in Miseq platform (Illumina, CA, USA). The analyses of the data that included 4813 clinically relevant genes revealed a novel homozygous PLEKHG5(NM_001265593.1):c.1600-2A>G mutation at the canonical splice acceptor site of PLEKHG5. This variant was not found in any healthy controls on GnomAD data. ${ }^{7}$ Online insilico analyzing tools had possible pathogenic predictions including evolutionary conservation and splicing impact (DANN score: 0.9906 and GERP score: 4,53) for the variant which could lead to the distribution of the splicing of intron 14 and cause none or less-functional protein. Three more PLEKHG5 gene splice-site variants other than the variant which is presented were reported in the Clinvar database and all of them were interpreted as likely pathogenic (rs1553174500, rs1553174566, rs144750655). However, clinical features associated with these splice-site variants were not reported. Recently, 10 cases with CMT and CMT-associated neuropathy were reported but none of the mutations were splice-site mutations. ${ }^{8}$ Just as the mutation detected in the patient was not found in HGMD Professional (HGMD version 2020.4) and Clinvar databases, and also it was not reported in literature screening. Confirmation and segregation analyses of the variant were completed via primers designed in-house for the next-generation sequencing system for the patient and the family. It documented the carrier status of the parents and a nonaffected sibling (Figure 2a). Since the loss of function mutations in the PLEKHG5 gene have been a well-known mechanism for the disease, all the variants in the splice region would produce the disruption of the splicing.

The interpretation of the scores in splicing effect prediction tools showed that $\mathrm{A}>\mathrm{G}$ alteration likely disturbs normal splicing, as it denotes acceptor lost (MaxEntScan and ASPP(Alternative splice site programme)). Together, two in silico analyses above might imply a harmful effect on proper functioning of the splice acceptor site of PLEKHG5 around c.1600-2A $>\mathrm{G}$ alteration. This variant was considered causative for the disease based on clinical concordance, segregation analysis, in-silico analyses, and the fact that this variant was not previously reported. Criteria for interpretation of the variant; a null mutation causing loss of function as it was found on the variant acceptor splice site (PVS1), according to the low frequency (PM2), and pathogenic 


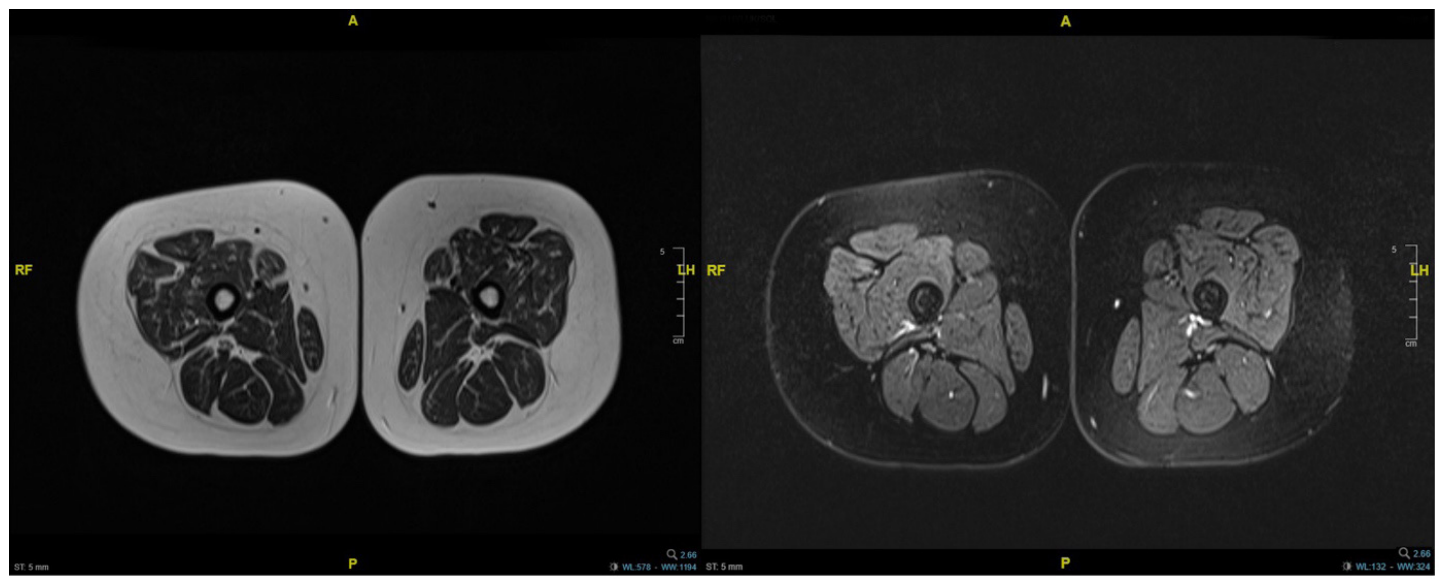

Figure 1: Lower-limb MRI showing Goutallier classification of 1 degree of fatty changes in both the anterior and medial compartment muscles.

predictions (PP3) for the variant with in-silico prediction tools; it was classified as likely pathogenic according to the Standards and Guidelines for the Interpretation of Sequence Variants. ${ }^{8}$ Figure $2 b$ shows the mutations of the proband and her family in IGV (integrative genomics viewer).

\section{DISCUSSION}

AR-CMTRIC related with compound heterozygous or homozygous mutations in the PLEKHG5 gene on chromosome $1 \mathrm{p} 36$ was reported previously.,4 In the present case, a novel splice site mutation was detected, in which a null variant led to a truncated less or non-functional protein. The variant was replaced in the RhoGEF domain (a guanine nucleotide exchange factor for Rho protein) of the PLEKHG5 protein, which contained several reported pathogenic variants. A lack of functional protein from both alleles of PLEKHG5 was explanatory for phenotypes

Genomic Position: Chromosome 1: 6530946

PLEKHG5(NM 001265593.1) Splice site

a.

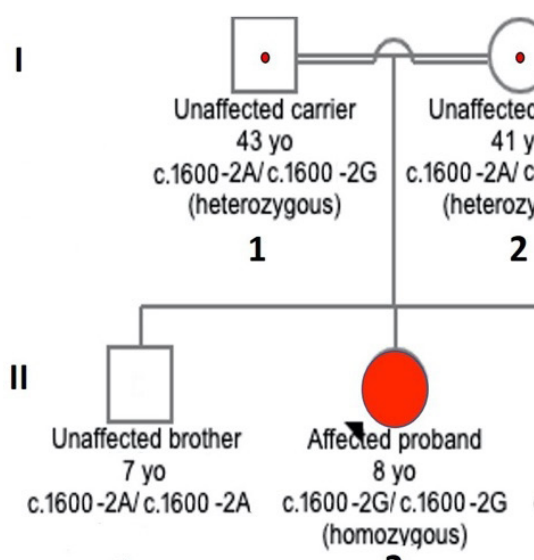

b.

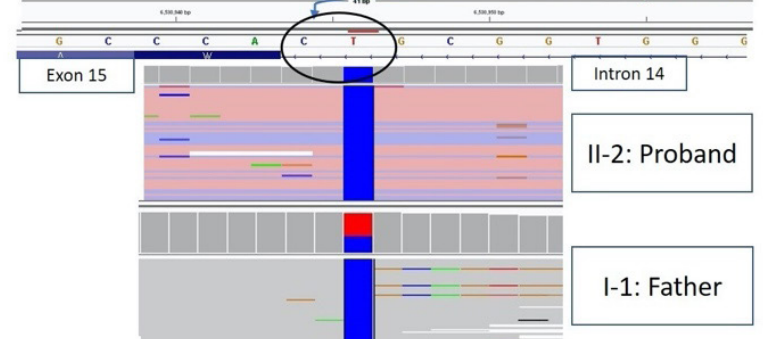

2
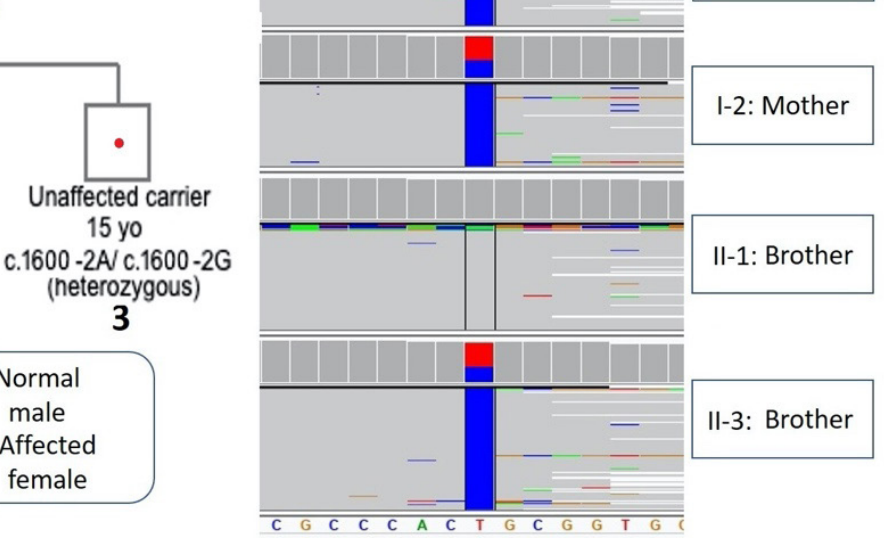

Figure 2a. Pedigree of the family $\mathbf{2 b}$. shows the mutations of the proband and her family in IGV (integrative genomics viewer) 


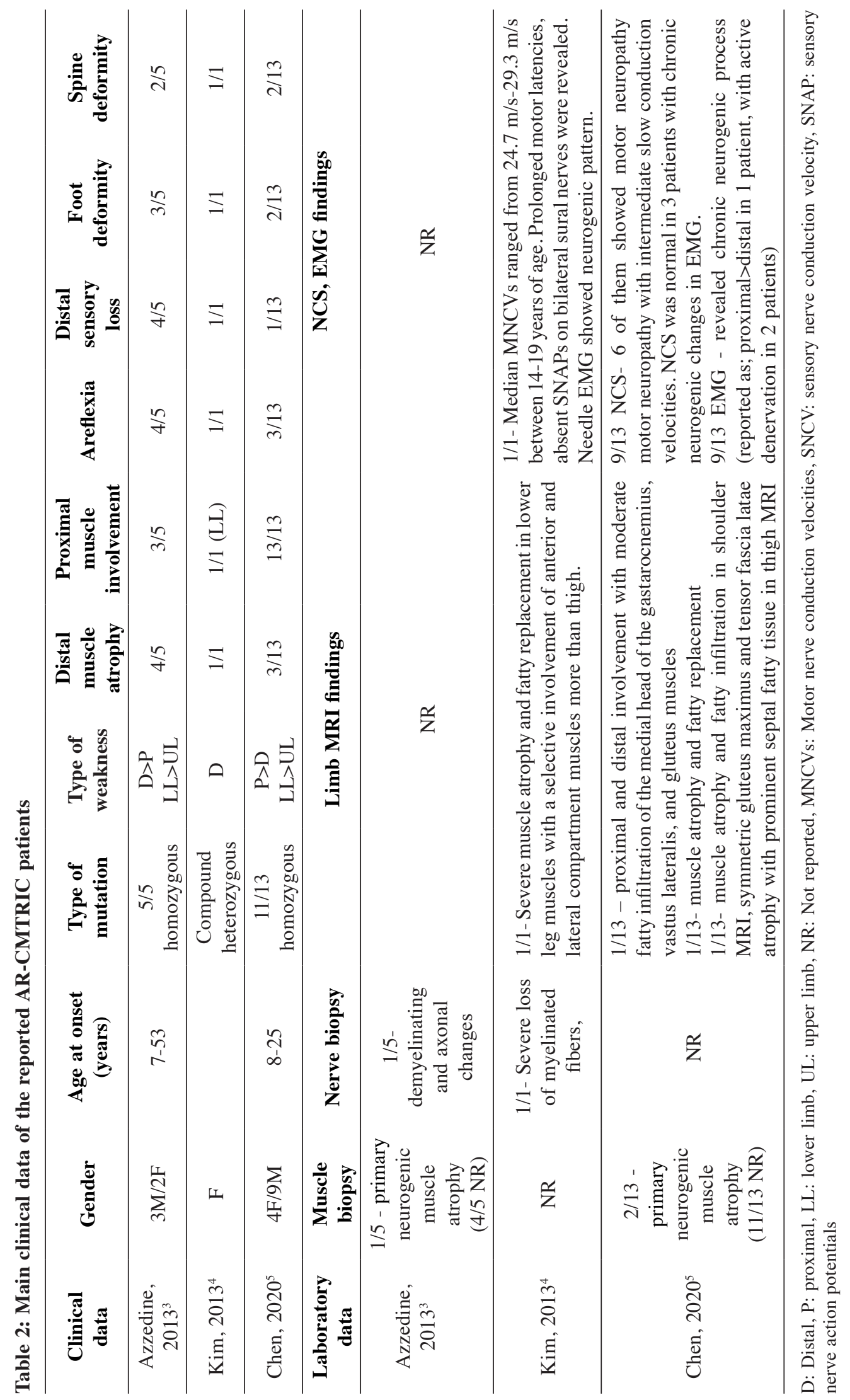


documented in laboratory investigations, including electrophysiological, and imaging findings. There is no healthy Turkish population data for the variant reported in this case, but segregation of the variant was demonstrated by a family study. Moreover, several researchers reported that mutations in the PLEKHG5 gene cause 2 different phenotypes, such as CMT disease, recessive intermediate $\mathrm{C}$, and spinal muscular atrophy, distal, and autosomal recessive CMT. ${ }^{2,48}$ Azzedine et al. were the first to experimentally design a mouse model lacking functional PLEKHG5, which mimicked the phenotypes observed in human patients. ${ }^{3}$ The main characteristics of the reported patients are summarized in Table 2 .

On admission, the case had weakness prominently in limb girdle muscle which was reported in almost all cases. ${ }^{8}$ Distal sensory loss was reported in almost $1 / 3$ of the patients. All of the patients had distal muscle weakness, predominantly in the lower limbs. On the contrary, weakness in the proximal muscles of the lower extremities was prominent in the patient in the current study. However, the presence of areflexia, distal muscle atrophy, and the development of spinal cord and foot deformity were consistent with other defined cases.

Electrophysiological studies of patients showed prolonged motor latencies and their median motor nerve conduction velocities were slowed (appropriate for intermediate phenotype), the amplitudes of the sensory nerve action potentials were decreased or absent, and their conduction velocities were slowed in the affected nerves. Needle EMG showed muscle denervation in almost all of the patients. In the present case, intermediate polyneuropathy was detected with moderately but predominantly demyelinating features of the sensory and motor nerves at first examination, while later axonal features became evident with active and chronic denervation findings. ${ }^{5,9}$

Kim et al.reported the involvement of anterior and lateral compartment muscles predominantly on hip MRI. ${ }^{3}$ However, in the current study, the lower-limb MRI of the patient showed Goutallier classification of 1 degree of fatty changes in both the anterior and medial compartment muscles.

In conclusion, this mutation was not reported to date; however, mutations affecting splicesites were reported as likely to be pathogenic in the literature. This mutation was classified as a likely pathogenic variant via American College of Medical Genetics and Genomics criteria. Moreover, the PLEKGH5 mutation in the patient herein showed clinical and electrophysiological evidence of sensory nerve involvement, which is a key point in the diagnosis of CMT. As reported previously, the EMG findings showed axonal sensory and motor polyneuropathy with some demyelinating features. These findings helped us to classify our case as CMT rather than spinal muscular atrophy associated with PLEKHG5 variants. Finally, this case showed a novel mutation, which will contribute to the literature in terms of genotype-phenotype correlation with clinical and electrophysiological findings.

\section{ACKNOWLEDGEMENTS}

All authors thank the patient and her family members for their participation in this study.

\section{DISCLOSURE}

Financial support: None

Conflict of interest: None

\section{REFERENCES}

1. Chhatriwala MK, Betts L, Worthylake DK, Sondek J. The DH and $\mathrm{PH}$ domains of Trio coordinately engage Rho GTPases for their efficient activation. J Mol Biol 2007;368:1307-20.

2. Maystadt I, Rezsohazy R, Barkats M, et al. The nuclear factor kappaB-activator gene PLEKHG5 is mutated in a form of autosomal recessive lower motor neuron disease with childhood onset. Am J Hum Genet 2007;81:67-76.

3. Azzedine H, Zavadakova P, Plante-Bordeneuve V, et al. PLEKHG5 deficiency leads to an intermediate form of autosomal-recessive Charcot-Marie-Tooth disease. Hum Mol Genet 2013;22:4224-32.

4. Kim HJ, Hong YB, Park JM, et al. Mutations in the PLEKHG5 gene is relevant with autosomal recessive intermediate Charcot-Marie-Tooth disease. Orphanet J Rare Dis 2013;8:104.

5. Chen Z, Maroofian R, Başak AN, et al. Novel variants broaden the phenotypic spectrum of PLEKHG5associated neuropathies. Eur J Neurol 2020; 28(4): 1344-55.

6. Ryan CS, Conlee EM, Sharma R, et al. Nerve conduction normal values for electrodiagnosis in pediatric patients. Muscle Nerve 2019;60:155-60.

7. Karczewski KJ, Francioli LC, Tiao G, et al. The mutational constraint spectrum quantified from variation in 141,456 humans. Nature 2020; 581(7809): 434-43.

8. Richards S, Aziz, N, Bale S, et al. Standards and guidelines for the interpretation of sequence variants: joint consensus recommendation of the American College of Medical Genetics and Genomics and the Association for Molecular Pathology. Genet Med 2015; 17; 405-23.

9. Saporta AS, Sottile SL, Miller LJ, Feely SM, Siskind CE, Shy ME. Charcot-Marie-Tooth disease subtypes and genetic testing strategies. Ann Neurol 2011;69:2233. 